\title{
Identificação dos Tipos de Jogadores para a Gamificação de um Ambiente Virtual de Aprendizagem
}

\author{
Thiago Henrique Flores, Ana Carolina Tomé Klock, Isabela Gasparini \\ Departamento de Ciência da Computação - PPGCA e PPGECMT \\ Universidade do Estado de Santa Catarina (UDESC) - Joinville, SC - Brasil \\ \{thiagohef, actklock\}@gmail.com, isabela.gasparini@udesc.br
}

\begin{abstract}
The gaming industry is growing and, from that, different forms of the same applications in the educational and business fields have been used. The gamification came from digital media industries, and can be defined as the use of game elements in contexts unrelated to games, using the process and the game mechanics to engage users and solve problems. In gamification, users can be classified in several ways, and among them, they can be classified by their player types, which is one of the ways to classify and understand the user's preferences. This paper aims to identify the player types and monitor students' interaction in a Virtual Learning Environment, to analyze which game elements engage each student in the teaching-learning process.
\end{abstract}

Resumo. A indústria dos jogos vem crescendo e, a partir disso, diferentes formas de aplicações dos mesmos nos meios educacionais e empresariais vêm sendo utilizadas. A gamificação surgiu nas indústrias das mídias digitais, podendo ser definida como o uso de elementos de jogos em contextos não relacionados com jogos, utilizando o processo e a mecânica do jogo para envolver os usuários e resolver problemas. Na gamificação, os usuários podem ser classificados de diversas formas e, entre elas, pelo seu tipo de jogador, que é uma das formas que os jogos utilizam para classificar e entender as preferências dos usuários. Este artigo tem o objetivo de identificar os tipos de jogadores e monitorar a interação do aluno em um Ambiente Virtual de Aprendizagem, como intuito de analisar quais elementos dos jogos mais engajam cada aluno no processo de ensinoaprendizagem.

\section{Introdução}

A gamificação é o processo de pensamento e mecânica de jogo utilizado para envolver os usuários e resolver problemas [Zichermann e Cunningham 2011]. De acordo com Paffrath e Cassol (2014), a gamificação aplica técnicas inerentes da área de design de jogos em diversas situações cotidianas, utilizando-se diferentes tecnologias de acordo com o meio aplicado. Quando aplicada na área educacional, a gamificação pode ser utilizada como uma forma de promover a motivação e o envolvimento dos alunos [Lee e Hammer 2011].

Um sistema gamificado utiliza diversas técnicas de jogos para aumentar a confiança e fazer com que o usuário queira interagir com o sistema [Zichermann e Cunningham 2011]. Embora ainda existam poucos estudos empíricos, Domínguez et. al. (2013) identificaram alguns trabalhos demonstrando que alunos sujeitos à aprendizagem através de sistemas gamificados são mais motivados a realizar tarefas, o 
que se traduz em uma maior participação nas atividades propostas. Estes resultados são obtidos quando a gamificação é consistente com os princípios dos jogos, onde os desafios são proporcionais à habilidade do jogador e promovem a sensação de recompensa e de realização pessoal, evitando a ansiedade e o aborrecimento [Monteiro e Santos 2014].

Para promover tal experiência, a gamificação pode considerar a classificação dos tipos de jogadores e, com base nela, atender aos usuários de forma mais específica ao apresentar técnicas mais adequadas a cada tipo. Essas classificações descrevem os usuários de acordo com suas preferências e seu perfil de interação com o sistema, possibilitando uma previsão de como os mesmos se portam perante as situações propostas.

A partir do exposto, este artigo tem o objetivo de identificar e monitorar os tipos de jogadores dos alunos que utilizam a gamificação de um ambiente virtual de aprendizagem, o AdaptWeb ${ }^{\circledR}$. Assim, o conceito de gamificação e alguns termos relacionados são introduzidos na seção 2 e a seção 3 expõe diversas tipologias disponíveis para a classificação dos perfis dos jogadores. A seção 4 apresenta o AdaptWeb $^{\circledR}$ e a seção 5 demonstra a identificação dos tipos de jogadores. A seção 6 apresenta as técnicas de gamificação utilizadas com base nos tipos de jogadores e a seção 6 descreve as considerações finais do artigo.

\section{Gamificação}

Os jogos e suas tecnologias vêm transcendendo cada vez mais os limites tradicionais de seu meio, evidenciando a aplicação dos mesmos nas indústrias e redes de ensino. Tal fenômeno é definido como gamificação, que é o uso de elementos e design de jogos para melhorar a experiência e engajamento do usuário em aplicações e ambientes que não são jogos. A gamificação tem atraído o interesse de muitos pesquisadores por ser um instrumento para criar um maior engajamento e também para facilitar a colaboração [Deterding et al. 2011].

Atualmente, muitas áreas aplicam as técnicas de gamificação no intuito de melhorar o rendimento e desempenho das pessoas envolvidas nos processos. Entre essas áreas, a educação faz uso das técnicas de gamificação para aumentar a motivação, interação e satisfação dos alunos com as atividades propostas. De acordo com Braga (2006), cada vez mais as instituições de ensino empregam softwares educacionais como facilitadores do processo de ensino-aprendizagem, utilizando-os como alternativa para os métodos tradicionais. Os softwares educacionais, segundo Valente (1993), têm os jogos em sua composição para motivar e desafiar o aluno, podendo exigir do mesmo algumas habilidades específicas como destreza, comparações lógicas e raciocínio.

Entre os estudos realizados para auxiliar no sucesso da gamificação, pode-se destacar a classificação dos tipos de jogadores, pois a identificação e classificação dos alunos de acordo com seus interesses no sistema facilitam na escolha das técnicas mais adequadas para motivá-los.

\section{Tipo de Jogadores}

Na gamificação, todos os usuários são descritos como jogadores e cada um possui suas próprias caraterísticas e particularidades de interação com o sistema. Conhecer os tipos 
de jogadores e seus comportamentos pode auxiliar os desenvolvedores e responsáveis pelo design institucional dos cursos a inserirem diferentes técnicas de gamificação para motivar cada um desses tipos. A partir disso, foi realizado um levantamento bibliográfico para identificar os diversos estudos que caracterizam o comportamento dos usuários nos jogos.

Foram encontradas oito tipologias, analisadas e sintetizadas na Tabela 1, onde são identificados os autores, a base de estudo (i.e., o que foi analisado sobre o jogador para gerar a tipologia), o método de identificação (i.e., o instrumento de coleta utilizado para realizar a identificação), os tipos de jogadores definidos e o jogo estudado.

Tabela 1. Análise dos Tipos de Jogadores Encontrados

\begin{tabular}{|c|c|c|c|c|}
\hline Autores & Base de estudo & $\begin{array}{l}\text { Método de } \\
\text { Identificação }\end{array}$ & Tipos de Jogadores Definidos & $\begin{array}{c}\text { Jogo } \\
\text { Estudado }\end{array}$ \\
\hline [Bartle 1996] & Comportamento & $\begin{array}{c}\text { Observação do } \\
\text { usuário }\end{array}$ & $\begin{array}{c}\text { Killers, Explorers, } \\
\text { Socializers, Achievers }\end{array}$ & $\begin{array}{c}\text { Multi-User } \\
\text { Dungeon }\end{array}$ \\
\hline $\begin{array}{l}\text { [Lazzaro } \\
\text { 2004] }\end{array}$ & Comportamento & Questionários & $\begin{array}{c}\text { Easy Fun, Hard Fun, Altered } \\
\text { States, People Factor }\end{array}$ & $\begin{array}{c}\text { Não } \\
\text { especificado }\end{array}$ \\
\hline [Whang 2004] & Preferências & Questionários & $\begin{array}{c}\text { Single-oriented Player, } \\
\text { Community-oriented Player, } \\
\text { Off-real World Player }\end{array}$ & Lineage \\
\hline [Tseng 2010] & Preferências & Questionários & $\begin{array}{c}\text { Agressive Gamer, Social Gamer, } \\
\text { Inactive Gamer }\end{array}$ & $\begin{array}{c}\text { Jogos on-line } \\
\text { em geral }\end{array}$ \\
\hline $\begin{array}{l}\text { [Kallio et al. } \\
\text { 2011] }\end{array}$ & Comportamento & $\begin{array}{l}\text { Triangulação / } \\
\text { Questionários }\end{array}$ & $\begin{array}{c}\text { Social Mentalities, Casual } \\
\text { Mentalities, Committed Mentalities }\end{array}$ & $\begin{array}{c}\text { Não } \\
\text { especificado }\end{array}$ \\
\hline $\begin{array}{l}\text { [Stewart } \\
\text { 2011] }\end{array}$ & $\begin{array}{l}\text { Comportamento e } \\
\text { Preferências }\end{array}$ & $\begin{array}{c}\text { Análise } \\
\text { Conceitual }\end{array}$ & $\begin{array}{l}\text { Primários: Guardian/Achiever, } \\
\text { Rational/Explorer, } \\
\text { Idealist/Socialiser, Artisan/Killer } \\
\text { Secundários: Conqueror, } \\
\text { Manager, Wanderer, Participant, } \\
\text { Hardcore, Casual } \\
\end{array}$ & $\begin{array}{l}\text { Analisa a } \\
\text { literatura } \\
\text { existente }\end{array}$ \\
\hline $\begin{array}{l}\text { [Nacke et al. } \\
\text { 2013] }\end{array}$ & $\begin{array}{l}\text { Comportamento e } \\
\text { Preferências }\end{array}$ & Questionários & $\begin{array}{c}\text { Seeker, Daredevil, Survivor, } \\
\text { Mastermind, Conqueror, } \\
\text { Socialiser, Achiever }\end{array}$ & $\begin{array}{c}\text { Não } \\
\text { especificado }\end{array}$ \\
\hline $\begin{array}{l}\text { [Marczewski } \\
\text { 2014] }\end{array}$ & Comportamento & Questionários & $\begin{array}{c}\text { Socialiser, Free Spirit, } \\
\text { Philantropist, Achiever, } \\
\text { Player, Disruptor } \\
\end{array}$ & $\begin{array}{c}\text { Sistemas } \\
\text { Gamificados }\end{array}$ \\
\hline
\end{tabular}

Analisando a Tabela 1, pode-se visualizar que os estudos se baseiam no comportamento e nas preferências dos jogadores. No que diz respeito ao método de identificação do tipo de jogador, a maioria das pesquisas utilizou um levantamento qualitativo por meio de questionários. Apenas uma das pesquisas realizou uma análise conceitual, que se baseia na evolução de tipologias existentes na literatura. Outro fator que se pode destacar é que todas as tipologias possuem um tipo de jogador que prefere interagir com os demais (i.e., Socializer, People Factor, Community-oriented Player, Social Gamer, Social Mentalities) em sua composição, indicando que a socialização e interação com o grupo é algo essencial. Ainda, de todas as tipologias encontradas, a única voltada especificamente para sistemas gamificados é de Marczewski (2014), descrita na subseção a seguir. 


\subsection{Tipologia de Marczewski}

A tipologia de Marczewski (2014) classifica os jogadores em seis tipos distintos, com base nos tipos de jogadores definidos por Bartle (1996). Seu principal objetivo é proporcionar aos designers de sistemas gamificados uma visão sobre o perfil dos usuários para facilitar a escolha das técnicas de gamificação mais adequadas. A tipologia de Marczewski (2014) descreve os tipos de jogadores:

- Achiever: é um tipo de jogador motivado pelo domínio. Os achievers buscam aprender coisas novas e melhorar a si mesmos, estando sempre procurando por desafios a superar;

- Disruptor: é um tipo de jogador motivado pela mudança. Em geral, os disruptors querem quebrar as regras, agindo diretamente ou através de outros usuários para forçar uma mudança positiva ou negativa no sistema;

- Free Spirit: é um tipo de jogador motivado pela autonomia e auto-expressão. Os free spirits gostam de criar coisas e explorar o sistema;

- Philanthropist: é um tipo de jogador motivado por um propósito coletivo. Estes jogadores são altruístas e gostam de auxiliar e proporcionar conquistas aos outros usuários sem nenhuma expectativa de recompensa;

- Player: é um tipo de jogador motivado por recompensas. Os players fazem o que é necessário para coletar as recompensas existentes em um sistema e sempre buscam o melhor para si mesmos;

- Socialiser: é um tipo de jogador motivado pelos relacionamentos que o sistema proporciona. Os principais objetivos dos socialisers ao utilizarem um sistema gamificado é a interação com os outros e as ligações sociais.

Pode-se observar que cada tipo de jogador é motivado por alguma satisfação: os Achievers são motivados pelo domínio, os Disruptors são motivados pela mudança, os Free Spirits são motivados pela autonomia, os Philanthopists são motivados pelo propósito, os Players são motivados pela recompensa e os Socialisers são motivados pelos relacionamentos.

\section{AdaptWeb ${ }^{\circledR}$}

O AdaptWeb ${ }^{\circledR}$ (Ambiente de Ensino-Aprendizagem Adaptativo na Web) é um sistema de hipermídia adaptativo (SHA) que permite a autoria e a apresentação adaptativa de disciplinas oferecidas em cursos EaD [Gasparini et al. 2004]. Os SHA são sistemas que constroem um modelo com os objetivos, preferências e conhecimentos individuais de cada usuário e utilizam dessas informações durante toda a interação, a fim de se adaptar às necessidades particulares do mesmo [Brusilovsky 1996][De Bra 2003]. Partindo do princípio que cada usuário tem objetivos e conhecimentos singulares, os SHAs adequam sua forma de apresentação e de navegação para exibir apenas as informações mais relevantes para cada perfil [Brusilovsky 1996].

O AdaptWeb ${ }^{\circledR}$ realiza a adaptação para proporcionar a alunos de diferentes cursos a apresentação e navegação adequadas as suas preferências individuais. Essa adaptação é baseada em algumas características dos alunos: o conhecimento sobre os conceitos da disciplina, as preferências navegacionais e a formação. Outra característica do sistema é a possibilidade de ofertar uma mesma disciplina para mais de um curso. Por exemplo, a disciplina de Cálculo I pode ser oferecida para alunos com formações 
diferentes (e.g., cursos de Ciência da Computação, Engenharia Elétrica e Engenharia Civil), pois seu conteúdo é adaptado para cada curso [Moissa e Gasparini 2014].

\section{Identificação dos Tipos de Jogadores no AdaptWeb ${ }^{\circledR}$}

A identificação dos tipos de jogadores foi realizada com base na tipologia de Marczewski (2014) descrita na subseção 3.1, por ser a única voltada para sistemas gamificados e por indicar as técnicas de gamificação mais indicadas para cada tipo/perfil. Os tipos de jogadores foram identificados através da tradução do questionário definido por Marczweski (2014). O questionário possui 24 perguntas, sendo 4 perguntas para cada tipo, conforme Tabela 2. Essas perguntas devem ser respondidas conforme uma escala de Likert de 5 pontos (onde 1 equivale a "Discordo Totalmente" e 5 equivale a "Concordo Totalmente"). A escala de Likert, segundo Mendes e Dalmoro (2008), é um instrumento para mensurar a realidade sobre um objeto em estudo. Como resultado, o questionário retorna o percentual de cada tipo de jogador do aluno, definindo seu tipo predominante e os tipos secundários. Esse questionário foi implementado no AdaptWeb ${ }^{\circledR}$, onde todo aluno deve respondê-lo para poder interagir com o sistema.

Tabela 2. Questionário de Tipo de Jogadores de Marczewski.

\begin{tabular}{|c|c|c|c|}
\hline \multirow{4}{*}{$\frac{\sqrt{0}}{\sqrt[0]{2}}$} & $\begin{array}{l}\text { Eu gosto de fazer cursos apenas para } \\
\text { aprender coisas }\end{array}$ & \multirow{4}{*}{ 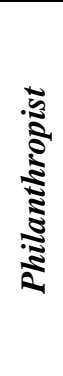 } & Eu gosto de ajudar as pessoas \\
\hline & $\begin{array}{l}\text { Eu geralmente realizo atividades até } \\
\text { aperfeiçoá-las }\end{array}$ & & $\begin{array}{l}\text { Eu gosto de voluntariar meu tempo para } \\
\text { ajudar a manter comunidades }\end{array}$ \\
\hline & $\begin{array}{l}\text { O caminho para conseguir algo é tão } \\
\text { importante quanto o objetivo final }\end{array}$ & & $\begin{array}{c}\text { Eu gostaria de contribuir com conteúdo } \\
\text { para comunidades on-line como a } \\
\text { Wikipedia }\end{array}$ \\
\hline & $\begin{array}{l}\text { Eu geralmente desisto se algo fica muito } \\
\text { difícil }\end{array}$ & & $\begin{array}{l}\text { Eu não gosto de compartilhar meu } \\
\text { conhecimento com os outros }\end{array}$ \\
\hline \multirow{4}{*}{$\frac{\sqrt{2}}{5}$} & $\begin{array}{c}\text { Eu sou um dos primeiros a adotar a uma } \\
\text { nova tecnologia }\end{array}$ & \multirow{4}{*}{ 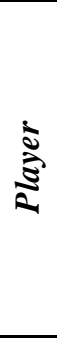 } & Ganhar é mais importante do que participar \\
\hline & $\begin{array}{l}\text { Eu me aproveito das falhas do sistema } \\
\text { para ganhar vantagem }\end{array}$ & & $\begin{array}{l}\text { Eu gosto de exibir as recompensas que } \\
\text { recebo }\end{array}$ \\
\hline & Eu geralmente tolero algo que não gosto & & $\begin{array}{c}\text { Eu fico feliz em doar itens para ajudar os } \\
\text { outros }\end{array}$ \\
\hline & As regras são feitas para serem quebradas & & $\begin{array}{c}\text { Eu gosto de competir para estar no topo } \\
\text { das tabelas de classificação }\end{array}$ \\
\hline \multirow{4}{*}{ 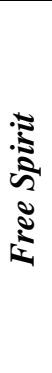 } & $\begin{array}{l}\text { A auto-expressão é muito importante para } \\
\text { mim }\end{array}$ & \multirow{4}{*}{ 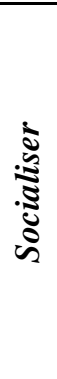 } & Eu uso de redes sociais regularmente \\
\hline & $\begin{array}{c}\text { Eu prefiro seguir uma história ao explorar } \\
\text { um ambiente }\end{array}$ & & $\begin{array}{l}\text { Eu prefiro conversar com as pessoas na } \\
\text { rede social em vez de segui-las }\end{array}$ \\
\hline & $\begin{array}{l}\text { Eu não gosto de ficar confinado por } \\
\text { muitas regras }\end{array}$ & & $\begin{array}{c}\text { Para mensurar o sucesso nas redes sociais, } \\
\text { o número de seguidores é a medida mais } \\
\text { importante }\end{array}$ \\
\hline & $\begin{array}{l}\text { Eu gostaria de encontrar surpresas } \\
\text { escondidas em jogos e filmes }\end{array}$ & & $\begin{array}{l}\text { Eu gosto de compartilhar conteúdo com } \\
\text { meus amigos e seguidores }\end{array}$ \\
\hline
\end{tabular}

Após responder o questionário, o aluno visualiza os seus percentuais em relação a cada tipo de jogador, conforme as respostas fornecidas. Os tipos de jogadores com maior percentual são chamados de predominantes e são destacados durante a apresentação, enquanto os demais tipos, chamados de secundários, ficam em segundo plano, conforme exemplifica a Figura 1. 


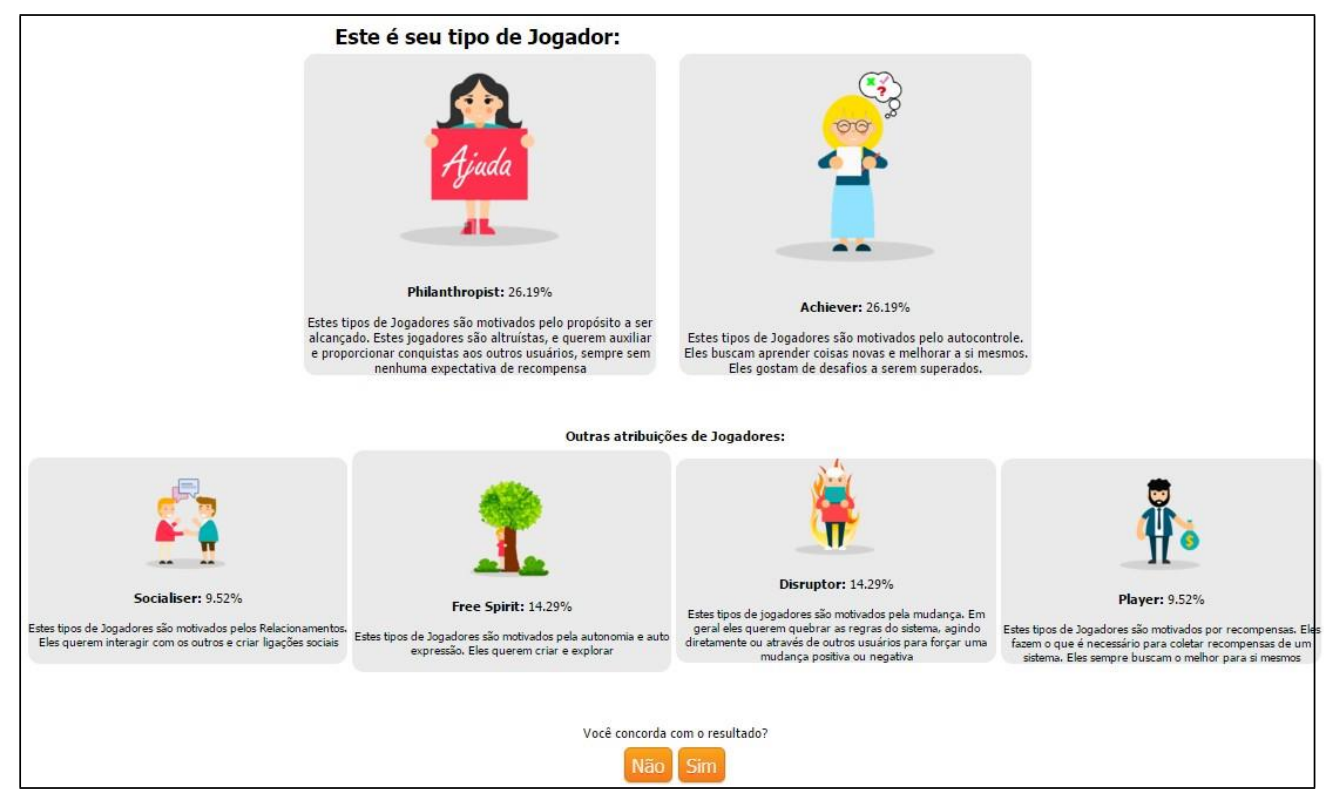

Figura 1. Exemplo de Resultado dos Tipos de Jogadores.

A partir dos resultados encontrados, o aluno pode concordar ou discordar do resultado. Caso discordar, o aluno é encaminhado para uma página onde pode definir qual sua afinidade com cada tipo de jogador com base em uma escala de 0 a 5 pontos (sendo 0 equivalente a "Não me identifico" e 5 equivalente a "Me identifico"), conforme Figura 2. Acredita-se que aqui o aluno possa fazer uma autorreflexão sobre suas características e, desta forma, o ambiente pode entender melhor cada indivíduo.

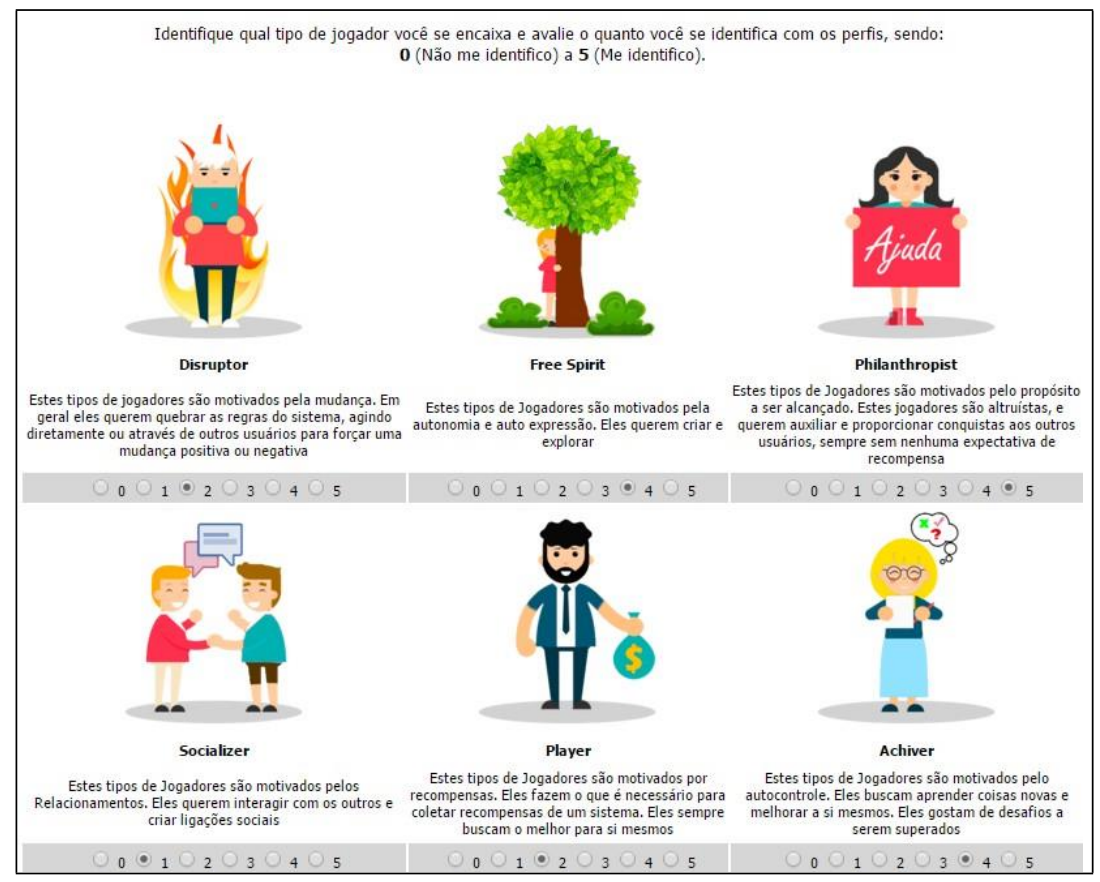

Figura 2. Página para que informar o grau de afinidade com cada tipo.

Conforme Figuras 1 e 2 o questionário está implantado no ambiente. Em um levantamento inicial realizado, 78 alunos responderam o questionário de levantamento de perfil e o questionário de tipo de jogador. Através dos resultados obtidos, tem-se: 
- Em relação ao sexo: 57 alunos (73\%) do sexo masculino e 21 alunas (27\%) do sexo feminino;

- Em relação à idade: 12 alunos $(15 \%)$ com idades entre 16 e 17 anos, 41 alunos (53\%) com idades entre 18 e 19 anos, 14 alunos (18\%) com idades entre 20 e 21 anos, 8 alunos (10\%) com idades entre 22 e 23 anos e 3 alunos (4\%) com 26 anos ou mais;

- Em relação aos cursos de origem: 3 alunos (4\%) do curso de Licenciatura em Física, 22 alunos (28\%) do curso de Tecnologia em Análise e Desenvolvimento de Sistemas, 10 alunos (13\%) do curso de Bacharelado em Ciências da Computação, 8 alunos (10\%) do curso de Bacharelado em Engenharia Civil, 6 alunos (8\%) do curso de Bacharelado em Engenharia Elétrica, 17 alunos (22\%) do curso de Bacharelado em Engenharia Mecânica e 12 alunos (15\%) do curso de Bacharelado em Engenharia de Produção e Sistemas;

- Em relação aos tipos de jogadores: os predominantes foram Achiever (21\%), Free Spirit (19\%), Philanthropist (19\%), seguidos pelos tipos Socialiser (16\%), Player (14\%) e Disruptor (11\%).

\section{Gamificação no AdaptWeb ${ }^{\circledR}$}

A partir dos resultados obtidos na seção 5, algumas técnicas foram implementadas no sistema: pontos de experiência, níveis, desafios, conquistas, ranking, regras, pontos de carma, desbloqueio de conteúdo e ranking infinitos, conforme as recomendações propostas por Marczewski (2014). A Tabela 3 faz a relação entre os tipos de jogadores com as técnicas implementadas. As três últimas colunas descrevem as técnicas implementadas depois da análise dos tipos de jogadores a fim de possibilitar a avaliação da forma mais igualitária possível.

Tabela 3. Tipo de Jogadores de Marczewski x Técnicas Implementadas.

\begin{tabular}{|c|c|c|c|c|c|c|c|c|c|}
\hline \multirow[b]{2}{*}{ Tipo de Jogador } & \multicolumn{9}{|c|}{ Técnicas Implementadas no sistema } \\
\hline & 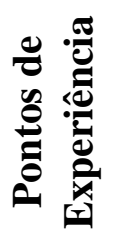 & $\frac{2}{2}$ & $\frac{0}{0}$ & ن & 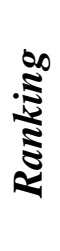 & 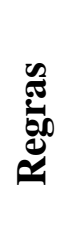 & 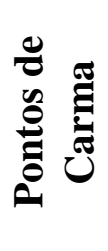 & 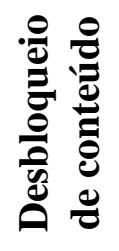 & 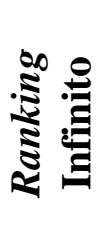 \\
\hline Achiever & & $\mathrm{X}$ & $\mathrm{X}$ & $\mathrm{X}$ & $\mathrm{X}$ & & & & \\
\hline Disruptor & & & $\mathrm{X}$ & & & $\mathrm{X}$ & & $\mathrm{X}$ & $\mathrm{X}$ \\
\hline Free Spirit & & & $\mathrm{X}$ & $\mathrm{X}$ & & & $\mathrm{X}$ & $\mathrm{X}$ & \\
\hline Philanthropist & & & & $\mathrm{X}$ & & $\mathrm{X}$ & $\mathrm{X}$ & & \\
\hline Player & $\mathrm{X}$ & & & $\mathrm{X}$ & $\mathrm{X}$ & & & & $\mathrm{X}$ \\
\hline Socialiser & & & $\mathrm{X}$ & & $\mathrm{X}$ & & $\mathrm{X}$ & & \\
\hline
\end{tabular}

Essas técnicas podem ser acessadas apenas quando o professor da disciplina define que a sua disciplina será gamificada. Assim, quando disponível, a gamificação aparece na página principal da disciplina, junto com as opções de acesso ao ambiente de aula, ao mural de recados, ao fórum de discussão e a ferramenta de análise de aprendizagem. Ainda, as técnicas de gamificação foram separadas em quatro abas: Perfil, Ranking, Moedas e Loja. 
A aba "Perfil" exibe as conquistas do jogador, tais como pontos de experiência, níveis, medalhas e um ranking que apresenta os cinco alunos com as melhores pontuações na disciplina, conforme Figura 3. As conquistas já alcançadas ficam destacadas, já aquelas ainda a serem alcançadas ficam esfumaçadas.

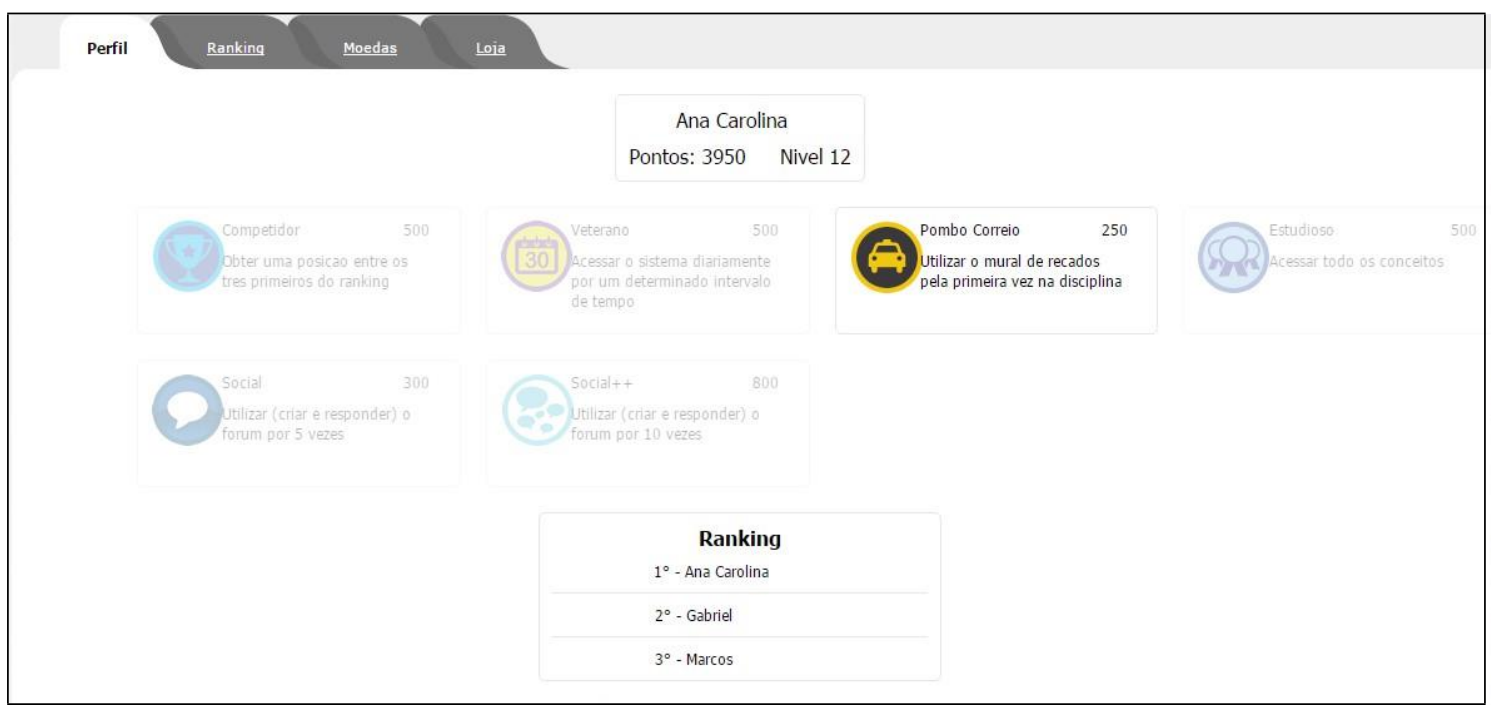

Figura 3. Perfil do Aluno no AdaptWeb ${ }^{\circledR}$.

A aba “Ranking" faz duas listagem dos alunos de forma decrescente conforme a maior pontuação e a maior quantidade de moedas adquiridas, ou seja, o aluno com mais pontos ou mais moedas fica na primeira posição. Quando o aluno acessa o ranking, sua posição fica destacada para facilitar a visualização.

$\mathrm{Na}$ aba "Moedas", o aluno pode transferir as moedas que conquistou para seus colegas. A quantidade de moedas que o aluno possui é apresentada na parte superior, seguida pela lista dos alunos e suas respectivas quantidades. Para efetuar a doação, o aluno seleciona um colega na lista e informa a quantidade de pontos a serem transferidos, conforme Figura 4.

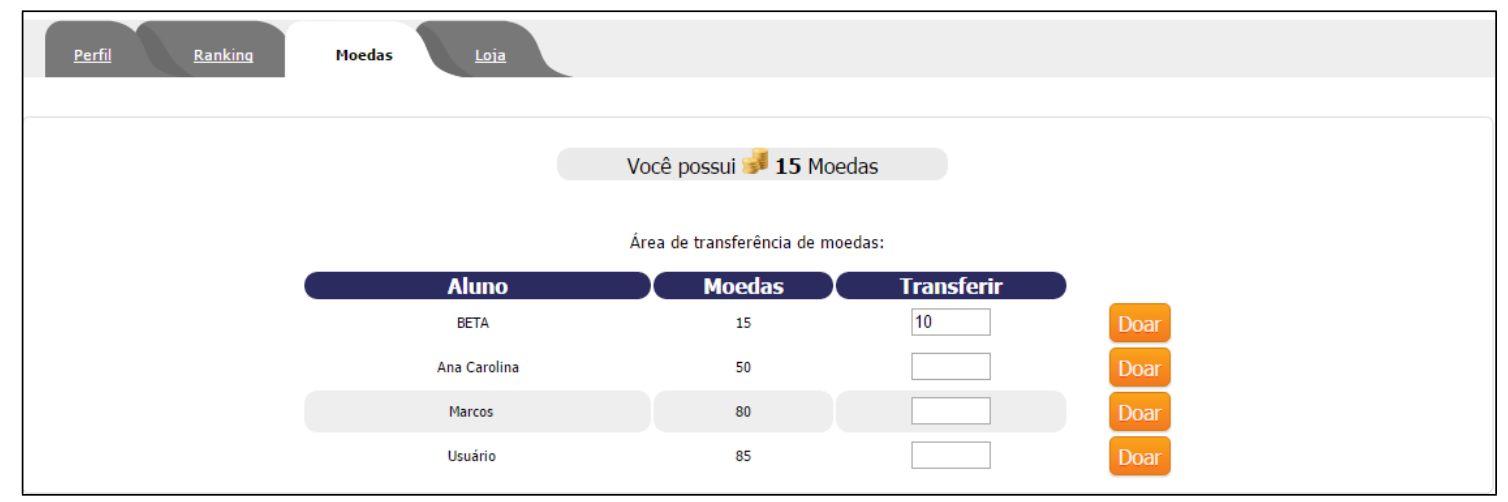

Figura 4. Transferência de Moedas entre alunos.

$\mathrm{Na}$ aba Loja, o aluno pode trocar suas moedas por presentes oferecidos pelo sistema, que são vantagens ao realizar certas atividades no sistema. Por exemplo, o aluno pode trocar 400 moedas por um presente que lhe permite obter 10 pontos a mais a cada exercício respondido corretamente. Esses presentes são comprados na loja e incentivam os alunos a realizar trocas e a interagir com os outros alunos. 


\section{Contribuição da Identificação dos Tipos de Jogadores}

Tal estudo e implementação no ambiente nos mostra como cada estudante está interagindo. Esta identificação nos permite saber se os alunos interagiram com os elementos propostos para cada tipo de jogador.

O estudo nos permitirá descobrir quais são as influências que os elementos podem ter nos alunos durante o percorrer das disciplinas, seja na sua motivação, desempenho, interação e satisfação. Este tipo de identificação também permitirá que possamos futuramente fazer com o sistema possa se adaptar a cada tipo de jogador mostrando a cada um deles uma quantidade de elementos que possam melhorar seu desempenho durante cursos online.

\section{Conclusão}

Atualmente, a gamificação está presente em vários sistemas, pois motiva seus usuários, assim como os estimula o progredir e interagir. Existem diversos aspectos que devem ser considerados durante a gamificação de um sistema e, entre eles, o tipo de jogador é uma das características mais importantes. Tal identificação facilita o projeto, pois analisa as preferências de interação do jogador e oferece ao usuário uma quantidade de técnicas que ele pode interagir.

Com base nesses dados da fundamentação e os trabalhos encontrados, pôde-se construir a proposta de trabalho, conferindo as técnicas que precisam ser implementadas no módulo gamificado do AdaptWeb ${ }^{\circledR}$ e identificando um questionário proposto com vinte e quatro questões para verificar os tipos de jogadores dos usuários, conforme proposto por Marczewski (2014).

Tais técnicas definidas, foram implementadas e colocadas em um ambiente virtual de aprendizagem. Os próximos passos de trabalho é analisar o resultado das interações obtidas e o resultado do questionário de tipo de jogadores, para confrontar se o tipo de jogador encontrado interagiu com as técnicas que são mais adequadas as suas características de acordo com Marczewski (2014).

Como trabalhos futuros, tem-se o intuito de fazer com que o sistema do AdaptWeb ${ }^{\circledR}$, possa adaptar a área gamificada para cada tipo de jogador especifico, podendo oferecer aos alunos as técnicas que mais são adequadas a seu perfil, fazendo com que ele tenha uma melhor interação. Tal pesquisa nos proporciona essa visão de como poderá ser realizada essa adaptação do sistema a cada tipo de jogador.

\section{Referências}

ADAPTWEB. AdaptWeb ${ }^{\circledR}:$ Ambiente de Ensino-Aprendizagem Adaptativo na Web. 2013. Disponível em: <http://ead.joinville.udesc.br/adaptweb>. Acesso em: 05 mai. 2016.

BARTLE, R. Hearts, Clubs, Diamonds, Spades: Players who suit muds. Disponível em <http://mud.co.uk/richard/hcds.htm>. Acesso em: 05 mai. 2016.

BRAGA, M. Design de Software Educacional Baseado na Teoria dos Campos Conceituais. Universidade Federal de Pernambuco, 2006.

BRUSILOVSKY, P. Methods and techniques of adaptive hypermedia. User Modeling and User-Adapted Interaction, 6 (2-3), pp. 87-129. 1996. 
CUNHA, L. Modelo Conceitual para a Gamificação em Ambientes E-Learning e sua utilização no AdaptWeb. Universidade do Estado de Santa Catarina, Joinville, 2014.

DETERDING, S.; SICART, M; NACKE, L; O'HARA, K; DIXON, D. Gamification: Using Game Design Elements in Non-Gaming Contexts. 2011.

DOMÍNGUEZ, A. et al. Gamifying learning experiences: Practical implications and outcomes. Computers \& Education, v. 63, p. 380-392, abr. 2013.

GASPARINI, I.; PIMENTA, M.; PALAZZO, J.; AMARAL, M. Navegação e apresentação adaptativos em um ambiente de EAD na Web. In: Webmídia 2004, Ribeirão Preto. Anais, 2004. v. 1. p. 1-1.

KALLIO, K. P.; MAYRA, F.; KAIPAINEN, K. At Least Nine Ways to Play: Approaching Gamer Mentalities. Games and Culture, v. 6, n. 4, p. 327-353, 1 jul. 2011.

LAZZARO, N. Why We Play Games: Four Keys to More Emotion Without Story. In XEODesign, 2004.

LEE, J.; HAMMER, J. Gamification in Education: What, How, Why Bother? In Academic Exchange Quarterly, 2011.

MOISSA, B.; GASPARINI, I. Técnicas de Visualização da Informação para Analisar o Comportamento de Alunos em um Ambiente E-Learning. Computer on the Beach. 2014.

MONTEIRO, S.; SANTOS, F. Gamificação dos Trabalhos de Grupo no Ensino Superior: o caso do Instituto Politécnico de Leiria. 2014.

MARCZEWSKI, A. A player type framework for Gamification Design. Disponível em <http://www.gamified.uk/user-types/>. Acesso em: 16 nov. 2015.

NACKE, L. E.; BATEMAN, C.; MANDRYK, R. L. Brainhex: A neurobiological gamer typology survey. 2013.

PAFFRATH, R.; CASSOL, V. Gaming Abroad: o uso de Gamificação no projeto de um sistema para Apoio a Turistas. XIII Simpósio Brasileiro de Jogos e Entretenimento Digital - SBGames. 2014.

STEWART, B. Personality And Play Styles: A Unified Model. In Gamasutra 2011. Disponível em: <http://www.gamasutra.com/view/feature/6474/personality_and_play_styles_a_.php>. Acesso em: 03 mai. 2016.

TSENG, F.-C. Segmenting online gamers by motivation. Expert Systems with Applications, v. 38, n. 6, p. 7693-7697, jun. 2011.

VALENTE, J. Aprendendo para a Vida: O uso da Informática na Educação Especial. In: M. P. Freire e J. A. Valente (orgs). 2001.

ZICHERMANN, G.; CUNNINGHAM, C. Gamification by design: implementing game mechanics in web and mobile apps. 1st. ed. Sebastopol, Calif: O'Reilly Media, 2011. 